\title{
Eco-Friendly Power Generator Cum Fitness Analyzer
}

\author{
Sanni Kumar, Roop Pahuja* \\ Dr B R Ambedkar National Institute of Technology Jalandhar (Punjab), Jalandhar 144011, India
}

Corresponding Author Email: pahujar@nitj.ac.in

https://doi.org/10.18280/i2m.190102

Received: 17 Otcober 2019

Accepted: 29 December 2019

\section{Keywords:}

body temperature, body mass index, heart rate, dynamo, human exercisig power, fitness model, virtual instrument

\begin{abstract}
The modern challenge faced with the global energy situation is the growing energy demand and the strong dependence on unsustainable fossil fuels. Another concurrent issue is the adverse health and socio-economic implications of adult obesity. Human Power Generation, which uses metabolized human energy to generate electrical power, could potentially address both these challenges. This paper discusses design and development of a method of exercising on a bicycle to convert the mechanical rotational power of human peddling to useable electrical energy using a dynamo integrated charge controller that charges a battery. Also, during exercising the health parameters such as body temperature and heart rate are reliably monitored using wearable sensors and wireless embedded processer to analyze the fitness level of a person and issue health alarm to prevent mishap during exercising. Further, the data is wirelessly transmitted to remote user interface for monitoring, logging and analyzing the electrical power generation capabilities of a person along with health condition. Experimental proven results indicate that system is capable to generate electrical energy during cycling by young humans with typical average efficiency of $17 \%$ or more. The system model is a smart power-generating exercising mechanism that is attractive and effective to be used in homes, gyms, health clubs and other extraterrestrial environments as a source of low cost sustainable green energy cum fitness analyzer.
\end{abstract}

\section{INTRODUCTION}

The world's energy consumption is all time high with the demand continuously increasing. This situation brings up several challenges that need to be addressed $[1,2]$ as:

- Depletion due to finite availability of non-renewable energy sources, e.g. fossil fuels.

- Increasing population, especially in developing countries which lack resources for clean energy.

- Environmental pollution, e.g. with coal use in power plants.

- Global warming with the related climate changes.

- Adverse implications of use of non-renewable energy resources such as burning of fossil fuel.

- Powering new technological applications such as ultraportable electronics devices, mobile chargers, laptops, wearable devices, remote wireless sensor nodes etc. that require portable low power DC power sources.

- Innovating simple, affordable and eco-friendly alternative means of energy harvesting and generation.

These challenges have been the reason for much controversy in the developed world; however, recent investigations have also shown a much more basic challenge of non-availability of power in the less developed parts of the world. Data from the World Bank in 2016 estimated that about $12.65 \%$ of the world's population has no access to electricity with Indian population being $22.38 \%$ [3] especially in rural areas (Table 1).

Challenges seldom come one at a time; in parallel with the energy problem, much of the world is also facing health issues of overweight and obesity. The World Health Organization estimated that in $2016,39 \%$ of women and $39 \%$ of men in the world with age 18 and above were overweight [4]. Overweight and obesity are often associated with low levels of physical activity [5]. Obesity decreases mechanical efficiency refers to the ability of an individual to transfer energy consumed by external work and high intensity exercise performance [6]. Obesity is also associated with musculoskeletal structure changes, decreased mobility, modification of the gait pattern, and changes in energy expenditure for a given activity [5]. Being obese or overweight has serious implications on one's health. The American Heart Association recognizes the condition of obese to produce significantly higher risks towards high blood pressure, high blood cholesterol, diabetes, heart disease and heart stroke [4]. Physical activity such as walking, running, cycling etc. is recommended to reduce excess body weight, prevent body weight regain, and decrease the subsequent risk of developing health related problems [5]. A solution that can address these two great challenges to control obesity and generate eco-friendly electrical energy, is physical exercisisng and the efficient use of human power during exercising for power generation. The main source of body energy is metabolized from sugar, fat and the output is configured to either extract direct mechanical work or convert the work to useable electrical energy.

Human power has been instrumental in solving problems since many decades. Use of flywheels, hand cranks and pedal power were efficient means of coupling human power to different applications [7, 8]. Bio-mechanical energy of human muscular strength during exercising is harnessed to generate eco-friendly electrical energy to power engineered 
applications Today, human power has been used in many innovative applications as an alternative source of renewable green and low cost energy with no carbon foot-prints and ewaste. Idea of powering solely from human energy exists as a technical challenge. For example, the American Society of Mechanical Engineers (ASME) holds the Human Powered Vehicle Challenge (HPVC) competition annually for encouraging higher education students to construct and compete with single-driver prototypes powered by the driver alone [9]. Human power has also been found to be uniquely good at providing energy generation in isolated situations. Authors in paper [10], shows the development of handoperated axial flux generators which is useful for dismounted soldiers, search and rescue operation in case of natural disasters, relief workers in remote regions and field scientists. Localized generation of electricity by humans is an excellent method of micro-power generation and autonomous operation of low cost electronic devices. Theoretical analysis indicates that brisk walking motion can produce up to $5-8 \mathrm{~W}$, adequate for basic wearable computing [11]. Shu and Lien [12] provide a summary of current progress in piezoelectric generator technology that indicate active power generation capabilities of up to $8400 \mu \mathrm{W}$ and has future to be incorporated in biomechanical energy generators. Micro-electromagnetic generator also features the unique aspect of passive electricity generation.
The thought of using human energy as an alternative and renewable energy source is gaining popularity to the level that businesses has been formed around converting exercise equipment such as stationary bikes to electricity generators [13]. Pedal power generation has even been established at a commercial level with the presence of companies such as Green Micro gym which sells do it yourself plans and equipment with which anyone can build a human powered generator [14]. It has been seen that human power generation has multiple applications in modern society. It is useful when users are isolated in the case of natural disaster, military deployment or being in a remote area. It also provides easy to implement and relatively low cost design which is particularly useful in rural areas of developing nations where skill in operating equipment and investment capital is limited. Furthermore, human power allows for power generation to be done socially, removing the feeling of deliberate effort while increasing the power output significantly $[10,11]$.

Motivated by the idea of harnessing electrical energy from human power of exercising and analyzing fitness level of a person, the proposed system is designed, developed and tested in realtime. The paper is organized into four sections that discuss the overview of the work, system architecture and hardware, application virtual instrument software, experimental work and results.

Table 1. Scenario of availability of electricity to countries [3]

\begin{tabular}{ccc}
\hline $\begin{array}{c}\text { 'Rank' in least availability of } \\
\text { electricity access in 2009 (out of 87) }\end{array}$ & Country & $\begin{array}{c}\text { Percent of population with access } \\
\text { to electricity in 2016 (\%) }\end{array}$ \\
\hline 1 & Central African Republic & 0.4 \\
2 & Burkina Faso & 0.80 \\
3 & Liberia & 1.30 \\
4 & Burundi & 1.70 \\
5 & Mali & 1.80 \\
6 & Chad & 2.20 \\
68 & India & 77.63 \\
\hline
\end{tabular}

\section{WORK OVERVIEW}

Though the use of dynamo on a bicycle is an existing old concept [15], but this has made a comeback as an alternative means to generate electrical energy in an eco-friendly and cost efficient manner. This work extends this idea innovatively to harness the bio-mechanical energy during exercising on a bicycle into useable electrical energy and analyzes fitness level of the person during exercising as well. The proposed system has great utility over the existing human power concepts and systems $[9,10,13,14]$. The objective of this work is to design and prototype an eco-friendly power generator cum fitness analyzer. The purpose is to develop a method of exercising i.e. peddling a bicycle to convert the mechanical rotational power to electrical energy using a dynamo fitted on a bicycle and simultaneously access fitness status of a person while exercising using wearable body temperature and pulse rate sensor. The body health sensors are interfaced to the standalone $\mathrm{Wi}-\mathrm{Fi}$ enabled embedded controller. As a person exercises on a cycle, the controller acquires the bio-parameters of a person along with electrical voltage data and wirelessly transmits the digital data packet to a soft-instrument (virtual instrument) on a PC over a Wi-Fi network. The dedicated application software (virtual instrument) collects, analyses the data and displays the vital electrical, health parameters and fitness alarm on remote user interface.

The system has been successfully used to experimentally study and online monitor the power generating and fitness level of various subjects (persons) in the age group of 20-30 years. The results obtained during the preliminary experimental work compare electrical power generating capabilities of different persons (normal, athlete and obese person) doing exercising (cycling) and estimate efficiency of power generator along with the variation in health parameters and its correlation with body mass index. The performance of the working prototype of the system in generating electrical power during cycling and on-the-go fitness analysis is found satisfactory.

\section{SYSTEM DESIGN}

Figure 1 shows the block diagram of the proposed ecofriendly power generator cum fitness analyzer. The system consists of different hardware and software elements that are integrated to form an embedded system with remote user interface. The inputs to the system, on one hand is the peddling power of a human (subject) exercising on a bicycle to provide 
electrical power output and on the other hand the body health signals that indicate the fitness level of the person doing exercising. The system hardware as explained below consists of circuits and components to generate energy from human power during cycling and fitness sensors that are interfaced to Wi-Fi enabled embedded controller.

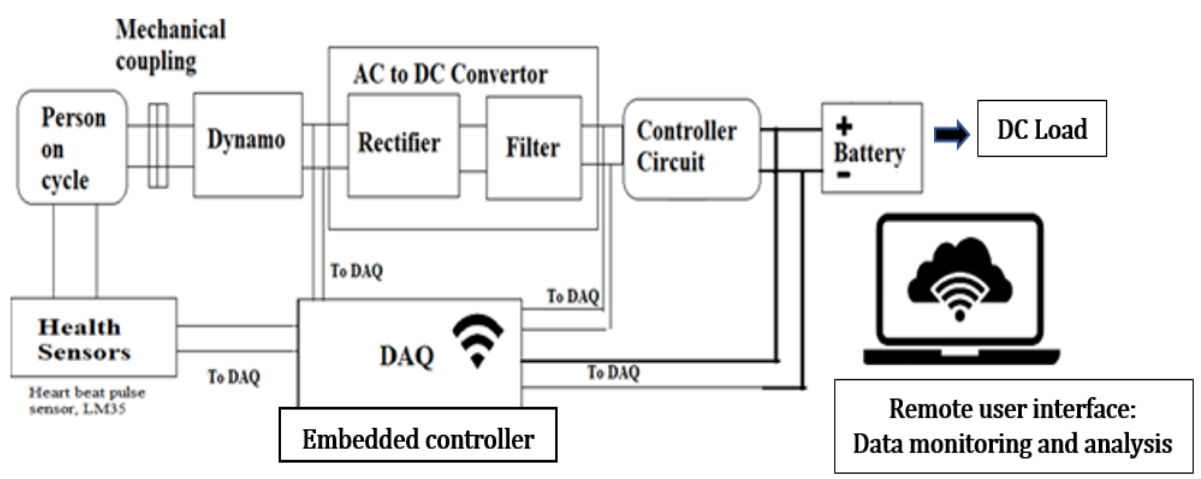

Figure 1. Block diagram of proposed eco-friendly power generator cum fitness analyzer

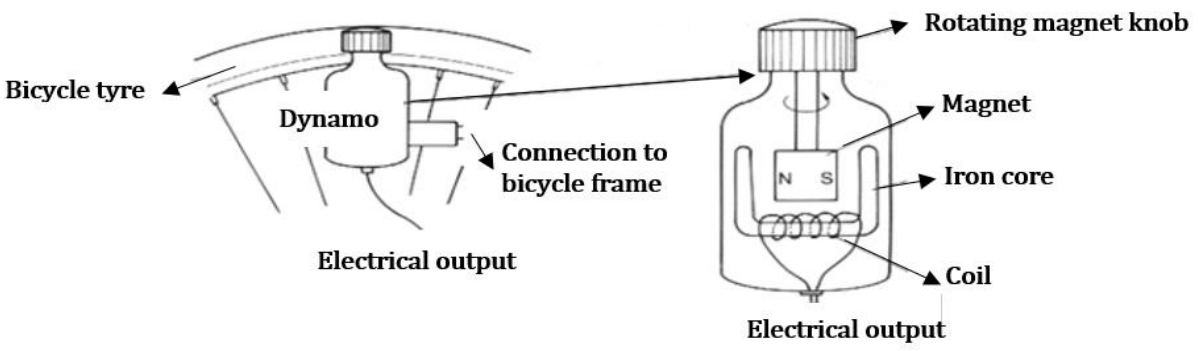

Figure 2. AC dynamo (alternator) roller coupled to a bicycle wheel [16]

(i) Human power generator: This is a primary unit that converts the bio-mechanical energy during cycling to rotational motion of the wheel into useable electric DC power that charges the battery to drive the load. It consists of an exercising bicycle fitted with AC dynamo (alternator), rectifier, charge controller and an electrical storage unit (battery). Figure 2 shows the connection of a bottle type sidewall dynamo [16] coupled to a bicycle wheel. The roller is placed on the sidewall of the bicycle wheel rim. When the bicycle is peddled, the wheel spins and the dynamo roller that is engaged to it rotates within the magnetic field of a permanent magnet. As the roller rotates, electrical output is produced based upon the principle of electromagnetic induction. AC dynamo basically consists of a rectangular coil mounted on a rotor shaft within the air gap of a permanent magnet. As the rotor rotates, the coil rotates perpendicular to the direction of magnetic field (B) of the dynamo. If the coil with plane area (A) rotates with an angular velocity $(\omega)$ in the magnetic field (B) then the flux $(\Phi)$ linked with the coil at any time t is given by Eq. (1)

$$
\Phi=\mathrm{B} \mathrm{A} \operatorname{Cos} \theta=\mathrm{B} \mathrm{A} \operatorname{Cos} \omega \mathrm{t}
$$

where, $\theta$ is the angle between the magnetic field vector $(B)$, area vector (A) given as $\theta=\omega \mathrm{t}$. Because of rotation of the coil, the magnetic flux through it changes and consequently voltage is induced in the coil. If the coil has $\mathrm{N}$ turns, then instantaneous value of the emf $(\mathcal{E})$ induced in the coil is given by the Eq. (2).

$$
\begin{gathered}
\varepsilon=-\mathrm{N} \mathrm{d} \varphi / d t=\mathrm{N} \text { B A } \omega \operatorname{Sin} \omega \mathrm{t} \\
\varepsilon=\varepsilon_{0} \operatorname{Sin} \omega \mathrm{t}
\end{gathered}
$$

where, NBA $\omega$ is the maximum or peak value of the emf, (Eo) and angular velocity $(\omega)$ of the coil of the dynamo depends upon the pedal velocity of bicycle [16].

The generated alternating voltage of a dynamo is connected to an external circuit by the means of slip rings. During exercising, as the peddling speed of a bicycle varies, the electrical output of the dynamo also varies in magnitude and frequency. Alternator, with typical rating of $12 \mathrm{~V}$, as used in the system prototype, provides variable AC voltage that is converted into DC voltage using full wave diode rectifier (DID4) and a capacitor (C1) as shown in Figure 3. The rectified dynamo voltage charges the $12 \mathrm{~V}$ battery through the buck type battery charge controller [17]. Battery charge controller is an automatic switching circuit that controls smooth charging of a battery at constant current from a variable DC source and prevents overcharging to ensure safe operation. The battery charge controller in the circuit provides the functions (i) to regulate the dynamo voltage to efficiently charge the battery to its rated value (ii) automatic cuts the input supply when the battery is overcharged (iii) prevents backward current to the source (iv) consumes low power.

The battery charge controller uses an adjustable voltage regulator IC (LM317) which provides line regulation (output voltage remains constant) and load regulation. The variable dynamo voltage is regulated to $12 \mathrm{~V}$ by the regulator of the battery charge controller. The charge controller circuit allows the regulated voltage to charge the battery if the battery voltage is less than $12 \mathrm{~V}$. In this condition, the regulator output provides the current that flows through the resistor R3 and diode D5 (IN4007) to charge the battery to its rated value. During the time battery charges, the Zener Diode (Z1) with typical value of breakdown voltage of $12 \mathrm{~V}$ does not conduct. 
When the battery voltage exceeds its rated voltage value of $12 \mathrm{~V}$, the current flowing through the battery is cutoff as Zener diode gets the sufficient breakdown voltage of $12 \mathrm{~V}$ to conduct. This allows the current to pass through the Zener diode to activate the low voltage NPN transistor (BD139). As the transistor (Q1) turns ON, the output current from the voltage regulator is grounded through the transistor. Thus, the transistor acts like a switch and cut-off the supply voltage from alternator by short circuiting the adjustable pin. The diode D5 in the circuit is used as a blocking diode to ensure that the current flows only in one direction so that the battery doesn't discharge when the alternator is not operational [17]. Further for analysis and display, dynamo rectified voltage and battery stored voltage are also interfaced to embedded controller using respective interfacing circuit.

(ii) Health sensors: To analyze the fitness of a person doing exercising, important body parameters such as body temperature and heart rate are being measured using temperature and heart beat pulse sensors. Readings of both these sensors are acquired using embedded controller and fused into fitness model to estimate the risk on health and generate health alarm.

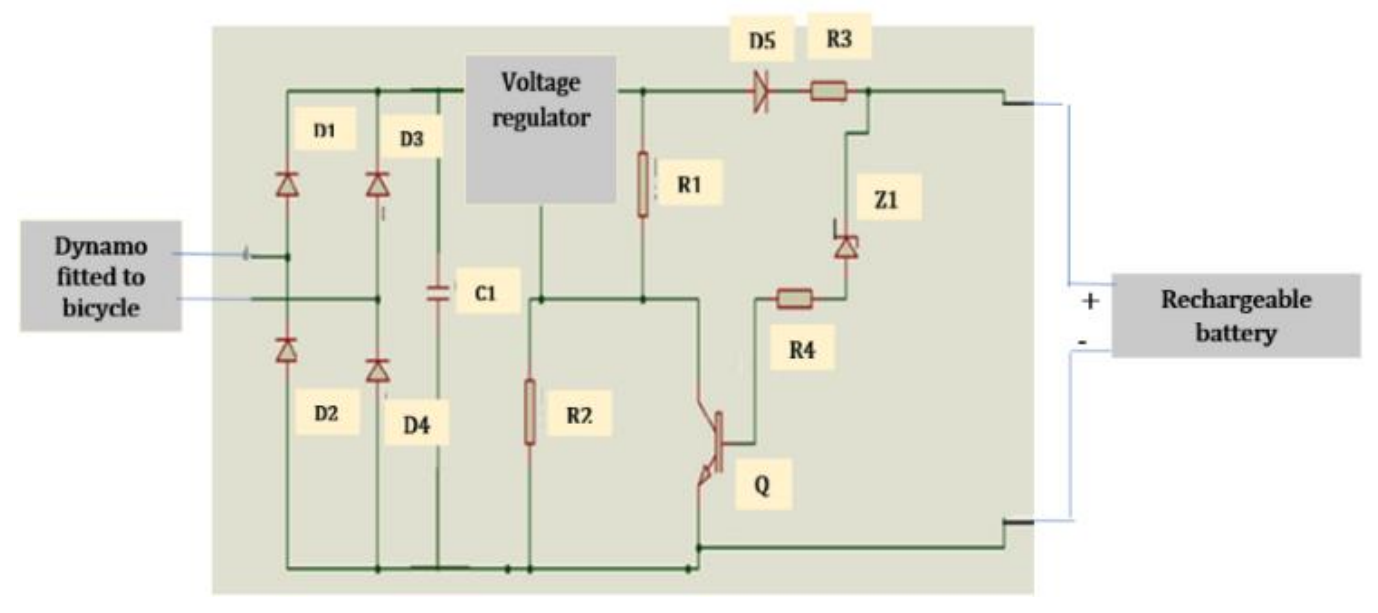

Figure 3. Circuit diagram of dynamo rectifier and automatic battery charge controller

Body temperature is measured using a popular LM35, low cost, precision grade, analog integrated temperature transducer. The device offers very good linearity, accuracy and constant sensitivity of $10 \mathrm{mV} /{ }^{\circ} \mathrm{C}$. The sensor has 3-pin terminals to provide input excitation signal of $5 \mathrm{~V}$ and get low power output voltage in the range 0 to $5 \mathrm{~V}$, directly compatible with embedded controller. For measurement of body temperature, the sensor is fixed near the nostrils of the person doing exercising. The temperature of the exhaled air as sensed by the sensor gives the measure of body temperature. In response to temperature of the exhaled air, the output voltage of the sensor varies linearly as given by the Eq. (4). The equation directly converts the measured sensor voltage readings in volts $(\mathrm{V})$ to body temperature value in degree centigrade $\left({ }^{\circ} \mathrm{C}\right)$ using a multiplying factor of 100 .

$$
\mathrm{V} \_\mathrm{TempS}=\left(10 \mathrm{mV} /{ }^{\circ} \mathrm{C}\right) \times \mathrm{T} \_ \text {body }
$$

Heart rate is measured using Photoplethysmography, a noninvasive, optical technique that detects changes in blood volume in peripheral blood vessels in relation to heart activity at various locations - including the earlobes, fingers, and toes. A PPG sensorr with in-build processing unit provides reliable detection of on-skin blood volume pulsations, detected during one cardiac cycle. This technique is popularly used in embedded medical application related to cardiovascular disease detection and monitoring [18]. A typical PPG based heart beat pulse sensor [19], used in the system prototype, consists of $660 \mathrm{~nm}$ bright red colour LED as light transmitter and photodiode as light detector placed opposite to each in through-beam (transmitted) configuration mode across the finger. The light source-detector pair is fabricated on a wearable band that is attached to the figure to detect the heart beat pulses. This PPG sensor works on the principle of modulation of red light by variation in oxygenated blood flow rate through the arteries at each heartbeat. The intensity of the transmitted red light is detected by the photodiode and processed by the signal conditioning unit to generate PPG waveform that indicates peak in the signal for each heartbeat. The sensor signal conditioning unit with band-pass filter minimizes the baseline wander, attenuates muscle noise, eliminates power line interference and amplifies the output signal in the range $0-5 \mathrm{~V}$ TTL level. When the heart contracts, it pumps oxygenated blood and the blood flows through the parts of body [19]. The bright LED light in PPG illuminates the nail. The oxygenated blood absorbs more of red light, so the intensity of red light detected by the detector is low henceforth, voltage output of the sensor varies from high to low level. The variation in the blood flow rate through the artery due to heart pumping action generates peaks in processed PPG signal waveform. For every cardiac cycle, apeak is genearted in processed PPG waveform. Continuously acquiring the analog output of the heart beat pulse sensor using embedded controller, detecting and counting the peaks in certain time interval using efficient peak detection algorithm heart rate in BPM (beats per minute) is estimated [18, 19].

(iii) Embedded controller and Wi-Fi communication module: For remote monitoring and analysis of human power generator data, a typical data acquisition (DAQ) and embedded controller device NI-MyRIO [20] with wireless communication capability is used. Figure 4 shows the circuit diagram to interface NI-MyRIO to system signals. Dynamo rectified DC voltage and battery voltage signals, through the attenuator circuits, are interfaced to analog input channels of ports MXPA and MXPB respectively of MyRIO channel selector. The attenuator circuit is a resistive voltage divider network with two series resistors, $\mathrm{Ra}=670 \Omega$ and $\mathrm{Rb}=330 \Omega$ respectively that converts the input voltage in the range of 0 - 
$12 \mathrm{~V}$ to output voltage across $\mathrm{Rb}$ in the range $0-4 \mathrm{~V}$ using linear mapping. Thus the voltage signals at MyRIO through the attenuator circuits gives the measure of rectified alternator voltage and battery voltage. Similarly, both the health sensors are also interfaced to MyRIO for analysis of fitness level. The low power analog output signals of three-pin body temperature LM35 sensor and heart beat pulse sensor are directly connected to analog input channels of MXPA and MXPB respectively of MyRIO and also powered from 5V DC excitation signal available at the ports.

The integrated wireless communication module within the embedded controller provides secure communication over WiFi network (IEEE 802.11g network standard) in $2.4 \mathrm{GHz}$ ISM band with maximum line-of-sight range of $150 \mathrm{~m}$. The sensor data as acquired and processed by the controller in a periodic manner is framed as data packet and wirelessly transmitted over the Wi-Fi network to LabVIEW based remote use interface on a personal computer.

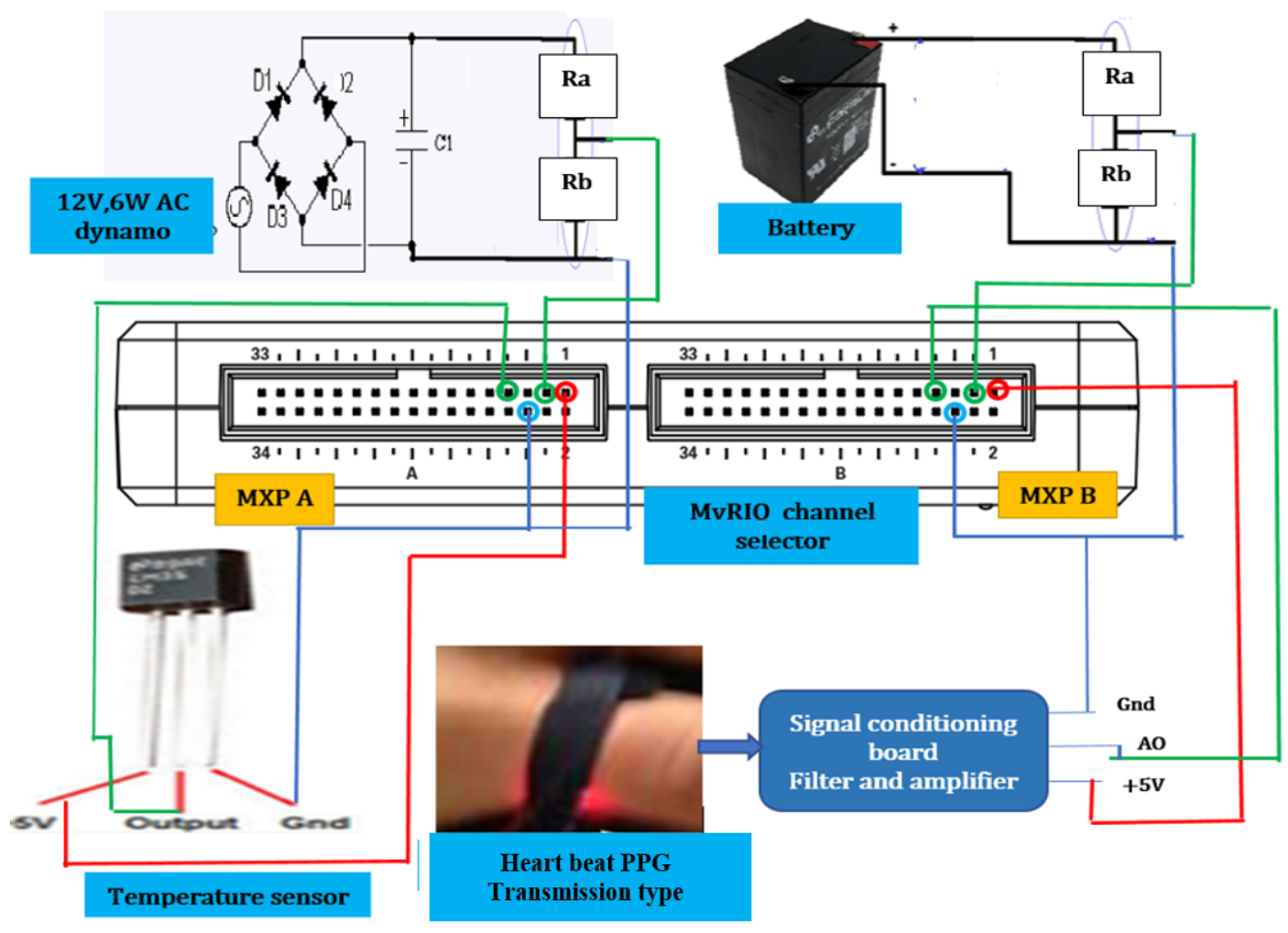

Figure 4. Circuit diagram to interface embedded controller (MyRIO) to the system signals

\section{HPG-FA SOFTWARE}

The system software named HPG-FA (human power generation and fitness analyser) is distributed virtual instrument programs on different processors designed on the platform of LabVIEW [21] with remote user interface. The soft-instrument defines the functions and features of the proposed system. HPG-FA software is implemented as an embedded virtual instrument code that runs on the processor of MyRIO and controls its functionality of data acquisition, processing and communication. The software also has remote virtual instrument on user terminal (PC) for wireless data acquisition, processing, logging, analysis along with data visualization on remote user interface. Figure 5 shows the functional elements of HPG-FA software. A brief description of software modules is given below:

(i) Embedded data acquisition and transmission: During the time a person exercises, the embedded virtual instrument samples the analog signals such as dynamo, battery, temperature and heart beat sensor voltages connected to MyRIO and transmits the processed data packet over Wi-Fi to remote user interface. The signals are continuously sampled with variable acquisition rate in a time frame of 10 s using sample-on-demand and N-sample data acquisition methods. Dynamo, battery and temperature sensor voltages are sampled at the rate of $10 \mathrm{~s}$ using sample-on-demand method and converted to rectified dynamo voltage $\left(\mathrm{v}_{\text {dynamo}}\right)$, battery voltage $(v)$ in volts and body temperature ( $\left.t_{\text {body }}\right)$ in degree centigrade using respective conversion formula as given by Eqns. (5), (6) and (7) respectively.

$$
\begin{gathered}
\mathrm{V}_{\text {dynamo }}=(1+\mathrm{Rb} / \mathrm{Ra}) \mathrm{V}_{1} \\
\mathrm{~V}_{\text {battery }}=(1+\mathrm{Rb} / \mathrm{Ra}) \mathrm{V}_{2} \\
\mathrm{~T}_{\text {body }}=100 \times \mathrm{V}_{\text {LM35 }}
\end{gathered}
$$

where, $\mathrm{Ra}=670 \Omega$ and $\mathrm{Rb}=330 \Omega$ are the attenuator circuit resistors and $V_{1}, V_{2}$ and $V_{L M 35}$ are the sampled volatge values of dynamo, battery and temperature sensor. Heart beat sensor is sampled continuously using $\mathrm{N}$-sample data acquisition mode at a fast rate of $20 \mathrm{~ms}$ time interval for $10 \mathrm{~s}$ to generate PPG waveform samples. The pre-processed data of all signals (dynamo voltage, battery voltage, body temperature and PPG waveform samples) is framed as data packet and wirelessly transmitted to remote user interface using in-built Wi-Fi communication protocol. The virtual instrument on the remote user terminal (PC) receives the data packet for further analysis and display in real time. 


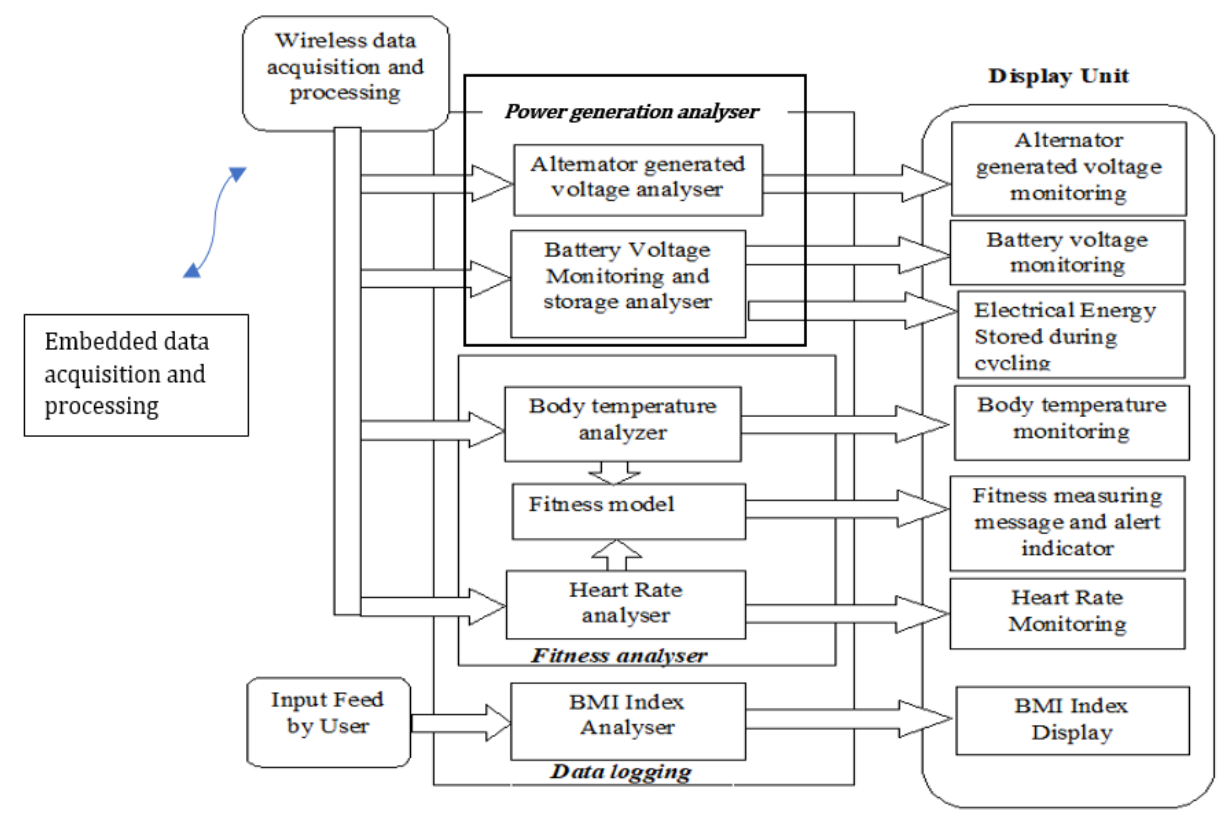

Figure 5. Functional elements of HPG-FA software

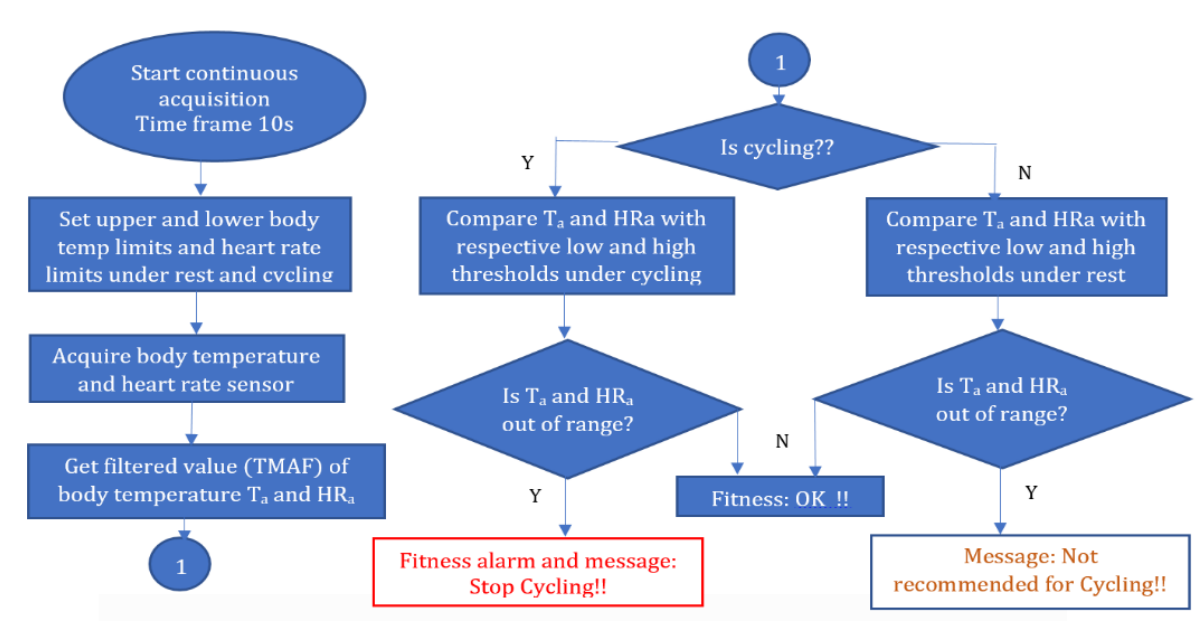

Figure 6. Flowchart of fitness check algorithm

(ii) Remote virtual instrument and user interface: HPG-FA software code on remote user terminal (PC) is a virtual instrument that synchronizes the operation of the system and performs function of data logging, analysis and visualization on user interface. Wireless data acquisition and processing program continuously acquires the wirelessly transmitted data packet on Wi-Fi network, extracts and log data to the system in real time with update rate of $10 \mathrm{~s}$. At each time instant, logged variables are analysed by subsequent programs as explained below:

(a) Power generation analyser: This software module processes the transmitted dynamo and battery voltage data at each instant of time using 6-point time moving averaging (TMAF) low-pass filter to indicate measured value of dynamo and battery voltage. It graphically monitors and updates the dynamo voltage and battery voltage to visualize the effect of cycling in generating electrical power that charges a battery. During cycling, battery voltage gradually increases from initial to final value. Dynamo generated voltage totally depends upon peddling capabilities of the person doing exercising i.e how strong and for how much time the subject is cycling. The increase in voltage value of the battery gives measure of increase in the energy stored in the battery. Hence the power generation capability of a person doing exercising is measured in terms of increase in battery voltage from initial to final value during the cycling time. Maximum energy stored in a battery $\left(\mathrm{BE}_{\max }\right)$ depends upon the charge stored in the battery given in terms of its ampere-hour rating $\left(\mathrm{B}_{\mathrm{C}}\right.$ in $\left.\mathrm{Ah}\right)$ and rated voltage $\left(V_{R}\right)$ and is given by Eq. (8).

$$
\begin{gathered}
\mathrm{BE}_{\max }(\mathrm{Ws})=(\text { Battery_charge }) \times \mathrm{V}_{\mathrm{R}} \\
=\left(\mathrm{B}_{\mathrm{C}} \times 3600 \mathrm{~s}\right) \times \mathrm{V}_{\mathrm{R}}
\end{gathered}
$$

(b) Fitness analyser: Fitness analyser measures important physiological parameters that effect the person's health such as body temperature and heart rate and fuses the health data in a fitness model to indicate fitness status of a person. Body temperature is a measure of body's ability to make and get rid of heat. Human body is very good at keeping its temperature within safe range, even when temperature outside the body changes. It is only during fever that body temperature deviates from the normal temperature. However, some variation in body temperature is also seen during exercising. For healthy person, during exercising, the body temperature remains near normal range. Continuous variation in body temperature during exercising is a matter of concern that is detected by the 
fitness analyzer. Another parameter that governs the overall health of a person is the functionality of heart, indirectly accessed in terms of heart rate. Many factors influence the heart rate including activity level, fitness level, stress, air temperature etc. Although there's a wide range of normal heart rate, an unusual high or low heart rate may indicate an underlying problem. Normal heart rate of adults under rest varies from 60 to 100 beats per minute (BPM). During exercising heart rate rises and varies in the range 100 to 170BPM. Accurate measurement of heart rate is important to detect abnormal variation of heart rate during exercising and timely warn the user about this to avoid any mishap. During exercising, heart rate is conveniently measured by noninvasive PPG sensor interfaced to embedded controller.

Temperature and heart rate data as acquired wirelessly from embedded controller is processed by temperature and heart rate analyser. Dynamic noise in temperature data is minimized using 6-point moving averaging low-pass filter to indicate body temperature value at each time instant of $10 \mathrm{~s}$. The preprocessed PPG waveform with distinct peaks, positive amplitude and no baseline drift as acquired from heart beat sensor is continuously dispalyed for 10 s. Each peak in PPG signal indicates one cardiac cycle counted as one heartbeat. Thus counting the number of peaks in PPG using peak detection algorithm as given below, heart rate in BPM is estimated. Further, at ecah time instant averaged value of heart rate is obtained using 6-point time moving averaging filter and dispalyed.

Peak detection algorithm for heart rate

IF (V_PPG) $)_{\mathrm{i}-1}>\left(\mathrm{V} \_\mathrm{PPG}\right)_{\mathrm{i}}$ AND $\left(\mathrm{V} \_\mathrm{PPG}\right)_{\mathrm{i}-2}$

AND ( $\mathrm{V}$ PPG) $\mathrm{i}-1>\mathrm{V}_{\text {th }}$

THEN COUNTER $=$ COUNTER +1

OTHERWISE COUNTER $=$ COUNTER +0

$\mathrm{BPM}=\mathrm{COUNTER} \times 6$

Based upon indicated filtered averaged body emperature $\left(\mathrm{T}_{\mathrm{a}}\right)$ and heart rate $\left(\mathrm{HR}_{\mathrm{a}}\right)$ measurements at each instant of time, fitness model performs the fitness check of a person during exercising. Figure 6 shows fitness check algorithm. If the body temperature or heart rate of a person continuously deviates from normal threshold ranges of each under rest or exercising condition, fitness alarm is activated. Also, the fitness message and audio alarm to continue or stop exercising is also generated.

(c) BMI analyser: Physical fitness of a person is quantified by a parameter BM1 (Body Mass Index) or Quetelet index. $\mathrm{BMI}$ is defined as ratio of weight (mass in $\mathrm{Kg}$ ) to the square of height $\left(\mathrm{m}^{2}\right)$ of a person given by Eq. (9). Measure of BMI is an attempt to quantify the amount of tissue mass (muscle, fat, and bone) in an individual, indicates proper balance of weight and height and categories the normal to obese state of a person.

$$
\mathrm{BMI}=\text { weight } /(\text { height })^{2}
$$

BMI analyser program takes user fed input about the weight $(\mathrm{kg})$ and height $(\mathrm{m})$ of a subject and calculates BMI. Based upon the BMI value, it also categories the physical fitness level of a person to indicate its underweight to obese state as per the defined standards [22] given as:

If $\mathrm{BMI}<18.5$ Then Message 'Person is underweight'

Else If $18.5<\mathrm{BMI}<25$ Then Message 'Person is normal weight'

Otherwise Message ' Person is obese'

\section{EXPERIMENTAL WORK AND RESULTS}

The system, as discussed above, is developed in compact and portable form to be attached to a bicycle. The dynamo is fitted to a bicycle wheel and the associated Wi-Fi enabled data acquisition hardware is hooked to the bicycle handle. The useable electrical energy generated by the dynamo charges the power source such as battery placed nearby to drive the external DC load. Further, the system has remote user interface on the PC/server (Figure 7) that wirelessly acquires the electrical and health signals of the person doing exercising, analyses the fitness and power generation capabilities of a person along with timely annunciation of fitness alarm to warn the person exercising under unhealthy conditions. The data of each person is logged for post analysis also. The salient function and features of the smart exercising system are:

- A simple/exercising bicycle is coupled to a dynamo to harness the human mechanical power of cycling to useable electrical DC power.

- For fitness monitoring of exercising person, health sensors are easily fixed to the person to reliably monitor body temperature and heart rate

- Supports wireless connectivity to home server or any PC over Wi-Fi network.

- Has a dedicated remote virtual instrument for data acquisition, analysis, logging and visualization.

- Has easy to use and informative soft user interface that supports real-time monitoring of dynamo and battery voltages on waveform graphs, digital meters for body temperature, heart rate, BMI, exercising time, electrical energy output and fitness alarm indicator.

- Analyses and keeps record of power generating capabilities of a person during exercising and health parameters under normal and during exercising mode.

- Has innovative feature to perform fitness check and activate fitness alert and voice activated fitness message to timely warn the user to stop exercising to avoid any health issues. Continuous exercising with irregular and high heart rate without indication can at times be fatal. Alerting on health issues is important to prevent any mishap.

The performance of the proposed economical eco-friendly system for power generation and fitness analysis is tested under real-time. The system is used by different persons (subjects) during relaxation and cycling/exercising for 15 minutes. The data for each person is online recorded, analysed and indicated on the remote user interface (Figure 7). Prior to online monitoring data of subjects doing cycling, preliminary information about weight and height of each subject are obtained through standard instruments. Body weight and height are manually measured by electronic weighing machine (range $0-200 \mathrm{~kg}$. resolution $0.2 \mathrm{~kg}$ ) and wall mounted measuring steel tape (range $2 \mathrm{~m}$, resolution $0.5 \mathrm{~cm}$ ) respectively. Initial validation check of the health sensors is also done to ensure the accuracy and consistency of sensor readings. Body temperature and heart rate of five persons are repeatedly measured five times using LM35 sensor and heart beat pulse sensor respectively on the virtual instrument of the set-up. The readings of both these sensors are compared and validated using the standard instruments such as clinical digital oral thermometer (range $35-42^{\circ} \mathrm{C}$ and resolution of $0.1^{\circ} \mathrm{C}$ ) and clinical portable pulse oximeter $( \pm 2 \%$ accuracy). Body temperature and heart beat pulse sensor readings are found to be highly correlated with the standard instruments, average 
correlation coefficient varying in the range $0.95-0.98$ thus ensuring the reliable measurement of health parameters in the proposed system prototype.

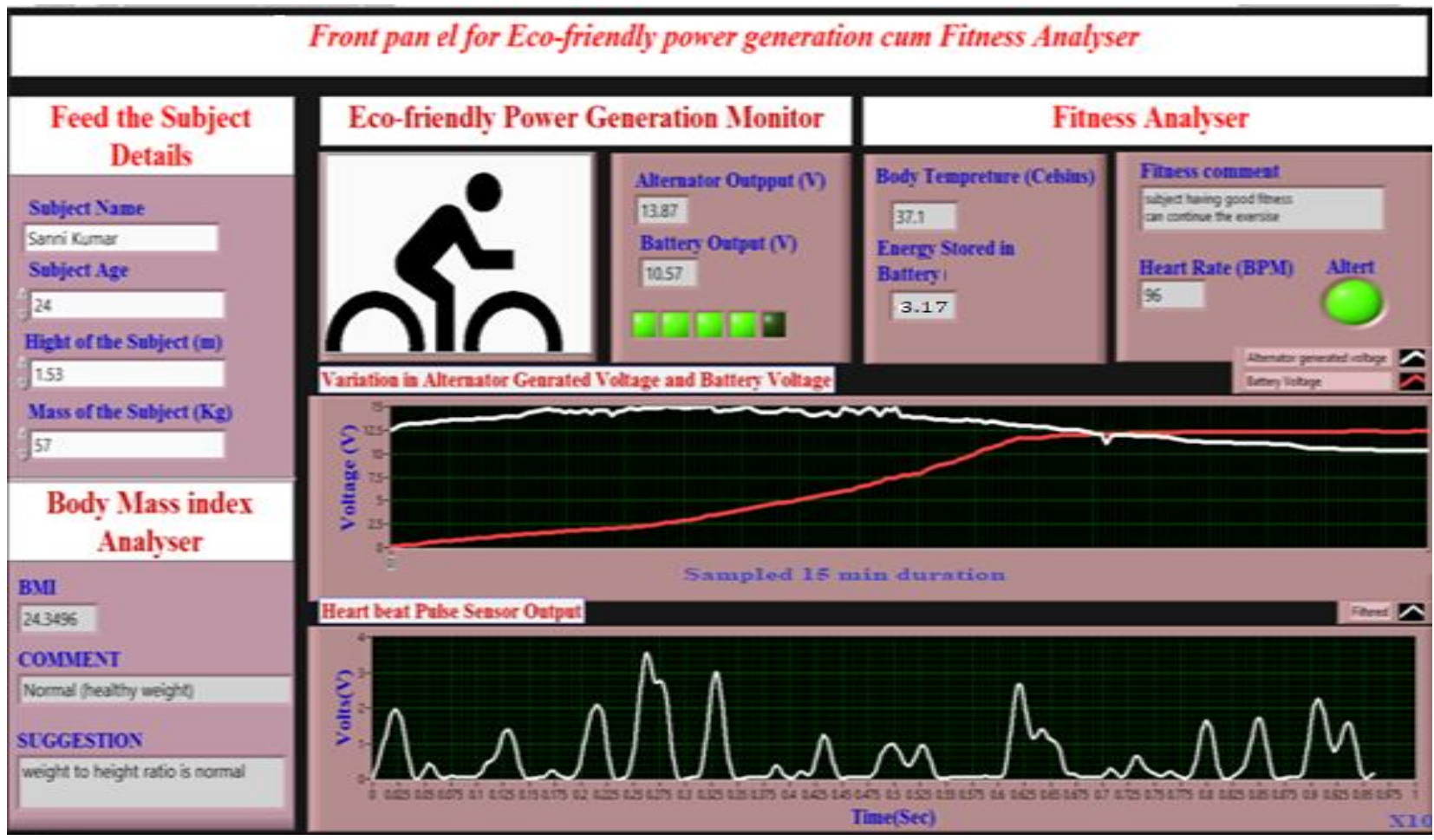

Figure 7. User interface showing power generation capabilities and fitness analysis of a person doing cycling for $15 \mathrm{~min}$

Figure 7 shows the graphical user interface of HPG-FA software indicating electrical power generation and health results of a person doing cycling. Prior to program execution, the subject details such as name, age, height and weight are entered through the user interface and on execution of the program, the system signals are captured, analysed and related results are displayed in real-time. The results are continuously updated on the panel at a fast rate of 10s. As indicated on the user interface, based upon the height and weight of a person as fed by the user, BMI value as indicated is 24.3 . This represents that the poerson has normal healthy weight and physically fitness level is OK. Time variation of alternator generated rectified DC voltage and battery voltage during cycling indicate the conversion of human cycling power to electrical energy. Initially, when the person starts cycling, dynamo voltage increases abruptly and battery is charged at a faster rate. After certain time interval, there is slight drop in instantaneous dynamo voltage as cycling speed reduces because of fatigue in legs. However, during continuous cycling for 15 minute, dynamo is eventually able to charge the battery to $12 \mathrm{~V}$. Thus the electrical energy generated is around 3.16 joules. Along with electrical signals, the user interface also simultaneously monitors the health parameters and fitness state of the subject during cycling. The heart beat sensor waveform displays the acquired PPG signal of 10s. PPG waveform is analyzed by peak detection algorithum to provide measure of heart rate as $96 \mathrm{BPM}$. The body temperature during exercising as measured is $32.1^{\circ} \mathrm{C}$. As both the body temperature and heart rate are within the prescribed normal ranges during exercising, the fitness level of the person is $\mathrm{OK}$. Fitness alert remains inactive and fitness message indicates 'Good fitness: Continue exercise'. If during exercising, the person is not passing the fitness check then fitness alert gets activated and voice activated warning message is also initiated to warn the user to stop exercising. This way the smart system keeps check on the health activity and power generation capability of the person doing exercising. Similarly, the system has been used by other subjects and data of each is logged for comparison.



Figure 8. Comparison of BMI, heartrate at rest, heartrate during cycling and energy stored in battery for different young persons doing cycling for $15 \mathrm{~min}$

Figure 8 shows the comparison of health and energy data of the different subjects doing cycling. The report indicates that the heart rate under rest of all physically fit normal weight person with BMI 18-25 varies within normal range of 72-90. During exercising, the heart rate of all persons increase to 130 BPM and above, which is more than the normal heart rate. However, for an obese person $(\mathrm{BMI}=35)$, there is slight increase in heart rate not only in rest condition but also during 
cycling. The heart rate shoots to 165 during cycling. As compared to the normal person, variation in heart rate of an athlete during exercising is not that high. Further, persons with same fitness level and age group, have the similar exercising pattern, capable of cycling efficiently to generate more or less same electrical power that charges the $12 \mathrm{~V}$ battery. During cycling for 15 minutes, a normal person generates and stores average energy of 3.1 joules in a battery. Moreover, the efficiency of the electrical power generator defined as the ratio of output electrical energy to input mechanical energy is variable. It depends upon human physical power of cycling, time duration and moment of inertia of cycle wheel. For a given exercising bicycle with moment of inertia $0.1 \mathrm{~kg} / \mathrm{m}^{2}$, normal young person cycling for 15 minutes with average speed of 60 rounds per minute, applies input rotational energy of 0.5 joules during cycling and generates average electrical energy of 3 joules. Hence, under ideal case, the typical efficiency of the power generator as estimated is $17 \%$.

This work has presented an innovative system with dual concept of generating electrical energy while exercising (cycling) along with on-the-go fitness analysis of a person for safe exercising. The proposed system is low cost and portable and can easily be hooked to an exercising bicycle for ecofriendly (green-energy) power generation and fitness analysis. Developed using a dynamo interfaced to embedded controller on Wi-Fi network along with health sensors and Windowbased user-friendly HPG-FA software (virtual instrument), the system provides remote online monitoring of energy generation and storage along with analysis of person's health and timely annunciation of fitness alarms during exercising. The fitness alarm warns the person doing over-exercising under unhealthy conditions and mitigate changes of casualties.

This system has great utility as alternative means of ecofriendly power generation that can easily be used to store energy to drive low power DC loads directly, such as LED lightning, charging units for electronic devices, laptops, mobile phones and low energy DC motors etc. Offering typical power generator efficiency of $17 \%$ or more, better than solar power generator, the proposed system is an ideal alternative to economic, eco-friendly, portable and maintenance free source of DC power with high level of utility. The system can directly be used in any home/building equipped with exercising cycle, gyms, health clubs, yoga centre, hostels, fitness clubs etc. as a source of free-cost energy generation and exercising cum health monitoring. Also, the need for exercise in space with low gravity makes the developed human power generator system a possible source for secondary power in extraterrestrial environments. In future, this proposed system can easily be upgraded with a few modifications to develop it as a ready-to-use commercial model for smart exercising cum fitness analysis and energy generation equipment.

\section{REFERENCES}

[1] Chen, Z.M., Chen, G.Q. (2011). An overview of energy consumption of the globalized world economy. Energy Policy, 39(10): 5920-5928. https://doi.org/10.1016/j.enpol.2011.06.046

[2] Wagner, L. (2014). Overview of energy storage technologies. Future Energy (Second Edition), Improved, Sustainable and Clean Options for our Planet, 613-631.

[3] The World Bank. Access of electricity (\% Population). http://data.worldbank.org/indicator/EG.ELC.ACCS.ZS/
countries/1W-USIN?display=default, accessed on July 2 , 2018.

[4] The World Health Organization. Overweight and obesity. http://www.who.int/gho/ncd/risk_factors/overweight/en/, accessed on June 20, 2012.

[5] Nantel, J., Mathieu, M.E., Prince, F. (2011). Physical activity and obesity: biomechanical and physiological key concepts. Journal of Obesity, 2011: 1-10. https://doi.org/10.1155/2011/650230

[6] Jabbour, G., Iancu, H.D. (2015). Mechanical efficiency improvement in relation to metabolic changes in sedentary obese adults. BMJ Open Sport Exercise Medicine, 2015(1). https://doi.org/10.1136/bmjsem2015-000044

[7] Frederiksen, W.A.C., Kim, S.Y. (1991). Hand cracker for nuts. United State Patent.

[8] Wilson, D.G. (1986). Understanding Pedal Power. Volunteers in Technical Assistance (VITA), Vieginia, USA.

[9] American Society of Mechanical Engineers. Human Powered Vehicle Challenge (HPVC) Engineering Competition.

http://www.asme.org/events/competitions/humanpowered-vehicle-challenge-(hpvc), accessed on Oct. 02, 2018.

[10] Bang, D.J., Ani, S., Polinder, H., Lee, J.Y., Moon, S.R., Koo, D.H. (2010). Design of portable axial flux permanent magnet machines for human power generation. IEEE International Conference on Electrical Machines and Systems, pp. 414-417.

[11] Paradiso, J., Starner, T. (2005). Energy scavenging for mobile and wireless electronics. IEEE Pervasive Computing, $4(1)$ : https://doi.org/10.1109/MPRV.2005.9

[12] Shu, Y.C., Lien, L.C. (2006). Analysis of power output for piezoelectric energy harvesting systems. Smart Materials and Structures, 15: 1499-1512. https://doi.org/10.1088/0964-1726/15/6/001

[13] Soldati, B.J., Szmuchrowski, L.A., Rocha, D.N., Correa, F.L.J., Sono, T.S.P., Vimieiro, C.B.S., Pinotti, M. (2010). Development of an adaptable system for a stationary bike to convert mechanical energy into electric power applied in indoor cyclism training. 6th International Conference on Technology and Medical Sciences, Porto, Portugal, pp. 95-99. https://doi.org/10.1201/b11330

[14] The Green Microgym. The world is made of energy, keep it flowing. https://www.thegreenmicrogym.com/thestory-of-the- upcycle-eco-charger/, accessed on Oct. 05, 2018.

[15] Francis, B. (2004). Bicycle based emergency battery charging system. U.S. Patent No. 6,717,280. http://www.freepatentsonline.com/6717280.html.

[16] Kullabs. Electric Motors and Bicycle Dynamo. https://www.kullabs.com/classes/subjects/units/lessons/ notes/note-detail/800, accessed on Oct. 2, 2019.

[17] ScienceDirect, Charge Controller - An overview https://www.sciencedirect.com/topics/engineering/charg e-controller, accessed on Oct. 2, 2018.

[18] Castaneda, D., Esparza, A., Ghamari, M., Soltanpur, C., Nazeran, H. (2018). A review on wearable photoplethysmography sensors and their potential future applications in health care. International Journal of Biosensors \& Bioelectronics, 4(4): 195-202. https://doi.org/10.15406/ijbsbe.2018.04.00125 
[19] Sunrom Technologies, Heartbeat Pulse Sensor - Analog Out, Datasheet, 2012.

[20] National Instruments. NI MyRIO-1900 User Guide and Specifications, $1-31$. http://www.ni.com/pdf/manuals/376047c.pdf

[21] Johnson, G.W., Jennings, R. (2011). LabVIEW
Graphical Programming. McGraw-Hill Education.

[22] World Health Organization, Body mass index - BMI, 2019. http://www.euro.who.int/en/health-topics/diseaseprevention/nutrition/a-healthy-lifestyle/body-massindex-bmi, accessed on Oct. 2, 2018. 